\section{A text-stimuli presentation manager for the IBM PC with ipsatization correction for response sets and reaction times}

\author{
ROSS BROUGHTON and NORMAN WASEL \\ University of Winnipeg, Winnipeg, Manitoba, Canada
}

IPSAPRO, an ipsative scoring program written for the IBM $P C$, aids in the detection and transformation of response sets that often contaminate rating scale and reaction time experiments. Response sets such as the tendency to use only extreme points of a rating scale or to work for speed over accuracy in reaction time experiments are removed in IPSAPRO by standardizing each subject's ratings or times against their own means and standard deviations. Ipsatization can be applied to existing data sets or take place automatically at the data collection stage in a textstimuli presentation manager that is provided with the program.

Response sets, or response styles, are reliable individual difference variables that are generated in part from artifactual features of measurement methods rather than the intended content of the stimulus (Nunnally, 1978). They attenuate the validity of research techniques because they tend to correlate very little or even negatively with the trait or process under study. Unlike other "nuisance" variables such as demand characteristics, which impose constant effects on all subjects, response sets exert systematic differential influences on subjects. Thus, response sets result from the interaction of subject and stimulus, which makes them harder to deal with. To control for response sets, information about a subject's particular style of responding is required.

Many response sets have been reported in the literature (cf. Cronbach, 1970; Nunnally, 1978). For instance, subjects differ reliably in the number of responses they give to open-ended questions (response productivity bias; Cronbach, 1949), the extent to which extreme responses are given on rating scales (extreme response tendency; Nunnally, 1978), the number of self-descriptors they endorse or check off as true (checking bias; Broughton, 1984; Strack, 1987; Wiggins \& Pincus, 1989), and the tendency to work for speed over accuracy in reaction time experiments (Cronbach, 1970). To neutralize these and other response set variables, a quantitative index of a subject's style of responding is required that can be used to weight responses. The ipsatization correction, first suggested by Cattell (1944), is such an index.

Ipsatized ${ }^{1}$ responses are standard scores calculated with individual subjects' means and standard deviations. As

Preparation of this article was supported by Social Sciences and $\mathrm{Hu}$ manities Research Council of Canada Grant 410-88-1322, awarded to Ross Broughton. We are grateful to James M. Clark, N. John Castellan, Jr., and a reviewer for their helpful suggestions on an earlier version. Correspondence concerning this article should be sent to Ross Broughton, Department of Psychology, University of Winnipeg, Winnipeg, Manitoba R3B 2E9, Canada. such, they reflect deviations from individual norms, in contrast to the usual standard score (e.g., $z, T$, etc.) that is distributed about group norms. When scores are ipsatized, they become effectively free of response sets that stem from the use of rating scales (e.g., Likert scales; dichotomous answer format; checklists, etc.) and reaction times.

Before one can conclude that a response set is in fact present in the data, and to what extent, a test for the shared common variance (SCV) between ipsatized and raw responses should be performed. This is easily accomplished by dividing the squared correlation between the two sets of scores by the product of their internal consistency estimates (coefficient alpha for interval data and the Kuder-Richardson formula 20 for dichotomous data). If the SCV is less than .90 , one should adopt the ipsatized scores (Nunnally, 1978). A more conservative approach is to use only the squared correlation between the two sets of scores and apply the same cutoff of .90. The latter descriptive index is provided in the present program; advice is given as to when to employ ipsatization on the basis of this cutoff, but the final decision to do so rests with the user.

As an alternative detection method, the effects of some response sets are easily found when raw data are subjected to factor analyses. True/false and checklist formats will usually give rise to a huge general first factor of positive loadings. However, when raw responses are ipsatized, as in the present program, this "response productivity bias" factor will disappear.

To illustrate the use of ipsative correction procedures, consider the following hypothetical situation in which 2 subjects with different response styles (extreme response tendencies) are compared. On a standard 7-place Likert scale, where " 1 " indicates "strongly disagree" and "7" indicates "strongly agree," Subject A tends to use the midpoints $(3,4,5)$ of the scale, while Subject B uses the extreme end points $(1,7)$. Although the 2 subjects share the same mean response (say, 4 ), they will differ widely in their standard deviations ( 1 and 3 , respectively). Although Subject A responds to stimulus one with a " 5 ", and Subject B responds with a "7," they are both conveying the same information (strong agreement), albeit with different communicative styles. In the case in which stimulus responses are summed (as in most ability and personality test scores) into, say, 10-item scale scores, a possible raw-score difference of 20 could occur between our 2 subjects, who are attempting to convey the exact same information. Thus, dramatically different interpretations could be made of patterns of results that are meant to be identical. Ipsatized transformations of these responses, however, would have yielded identical responses.

Despite their usefulness, ipsatization techniques are not available as procedural options in any of the major canned 
statistical packages such as SPSS $^{\boldsymbol{x}}$, SAS, BMDP, etc. Even the case-based statistics required to compute ipsative scores from within these programs, when available, are not easy to manipulate. The microcomputer versions of these packages make programming ipsative scores even more difficult.

In order to facilitate the use of ipsative scoring procedures, and to test for response set effects from within a data collection microcomputer environment, the program IPSAPRO was developed. In IPSAPRO, ipsatization can be applied to existing data sets or take place automatically at the data collection stage. This is accomplished from within a text presentation manager that permits the user to build tests or sets of stimuli and run them with ipsative scoring and millisecond timing features already built in.

\section{The Program}

IPSAPRO is written in two compiler versions of BASIC: (1) Power BASIC (formerly Borland's TURBO BASIC, now a product of Spectra Publishing ${ }^{2}$ ) and (2) Microsoft QuickBASIC. ${ }^{3}$ Power BASIC boasts more precise timing capabilities than its competitors. According to Spectra, at the software level, Power BASIC is capable of accuracy to $2 \mu \mathrm{sec}$ using Channel 2 of the IBM PC 8255 timing chip, which is consistent with the findings reported by Smith and Puckett (1984). We selected Power BASIC to allow users to exploit the potential of its microtimer function, especially given the low (55msec) level of resolution of the IBM BASIC timer function (Graves \& Bradley, 1987). However, given the many hardware timer contaminants known to exist in IBM PC systems (e.g., discrepancies among display raster scan

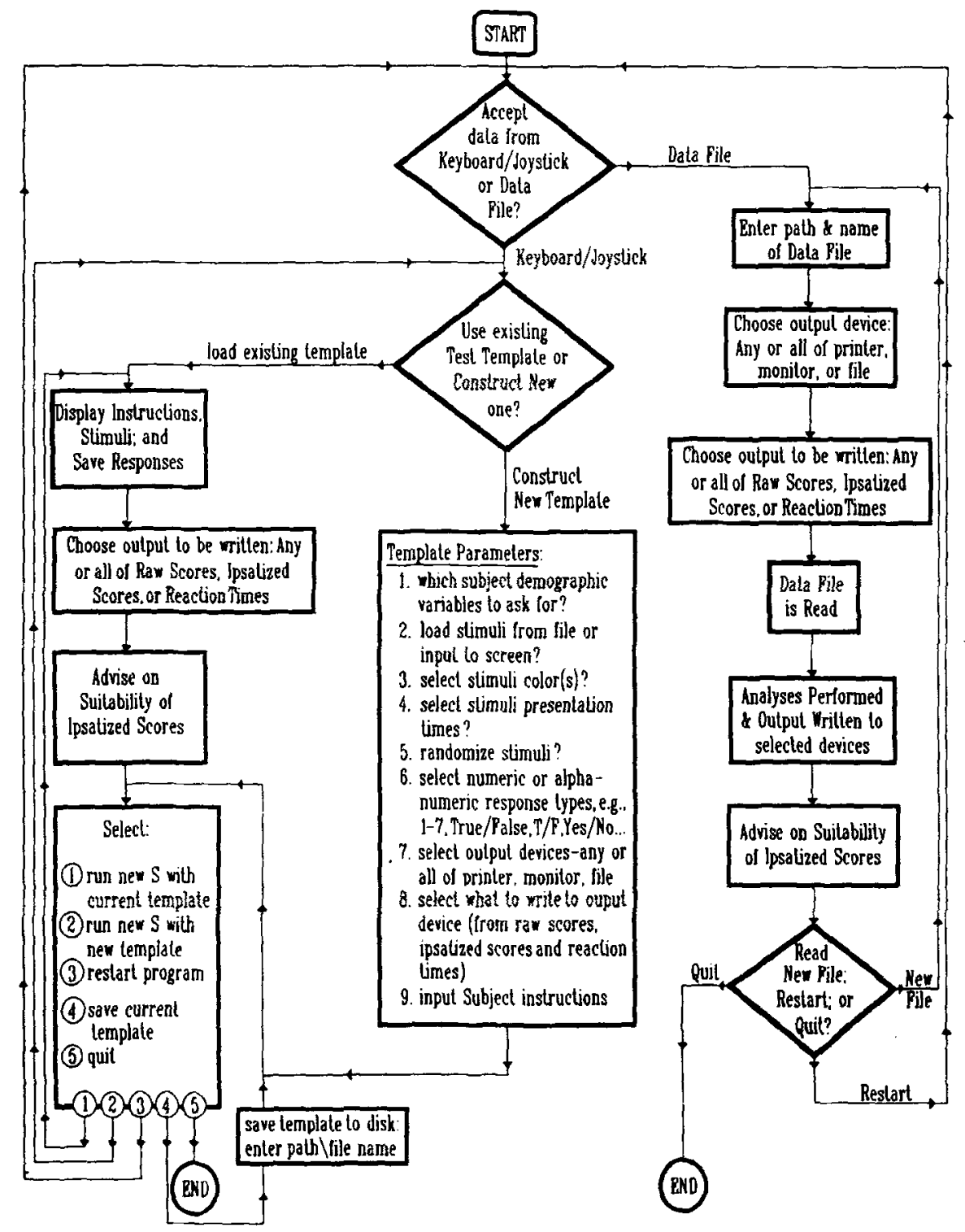

Figure 1. Flow chart for IPSAPRO. 
rates, keyboard latencies, etc.), those who require millisecond, or higher, precision will need to verify the Power BASIC microtimer (MTIMER) function on their systems. When a keyboard is to be used as an input device, high timer resolution is probably not possible. On this topic, Graves and Bradley (1987) advised users to expect keyboard accuracy only to within $5 \mathrm{msec}$, and this is after the mean latency error for individually tested keyboard keys has been removed (an operation available in the Power BASIC version of IPSAPRO). However, when the input device was a joystick, an average 0.55 -msec resolution was obtained, without transforming the times. For Microsoft QuickBASIC users, and/or those who do not require a millisecond resolution timer, a QuickBASIC (compiled and source code) version of IPSAPRO is also provided.

IPSAPRO is a menu-driven program that allows the user to either ipsatize existing data sets, or employ a textstimuli presentation manager to load or construct a testing template, or environment, to be used for running subjects. As is depicted in the flowchart in Figure 1, one can elect to follow one of three major program paths: (1) load an existing testing template, (2) construct a new one, or (3) bypass testing in favor of ipsative scoring of an existing data set. A testing template can be configured in such a way as to do all or some of the following: Ask for subject demographic information; load text stimuli from file or keyboard; randomize stimuli; select stimuli color(s); select stimuli presentation times or wait for subject response; time the reactions from onset of stimulus to onset of response (choose microtimer in Power BASIC version only); display a Likert scale (e.g., 1 through 7 , anchored with labels for lowest and highest values) or binary string scale (e.g., T/F, Y/N, etc., in which strings are converted to $0 \mathrm{~s}$ and $1 \mathrm{~s}$ ) for subjects to use in selecting responses; determine output devices (any or all of printer, monitor, or file); select scores to write to device (from raw scores, ipsatized scores, and reaction times); and input subject instructions from file or keyboard. When a template has been selected or constructed, the ex- perimenter may then proceed to run subjects, save the template, construct a new one, or restart the program.

\section{Availability}

Copies of source-code and object-code files of IPSAPRO are available from the first author via e-mail, bit-

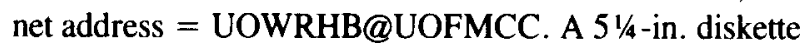
containing these files is also available from the first author for $\$ 3$, to cover materials and postage.

\section{REFERENCES}

Broughton, R. (1984). A prototype strategy for construction of personality scales. Journal of Personality \& Social Psychology, 47. 1334-1346.

Cattell, R. B. (1944). Psychological measurement: Ipsative, normative and interactive. Psychological Review, 51, 292-303.

Cronbach, L. J. (1949). Statistical methods applied to Rorschach scores: A review. Psychological Bulletin, 46, 393-429.

Cronbach, L. J. (1970). Essentials of psychological testing (4th ed.). New York: Harper \& Row.

Graves, R., Bradley, R. (1987). Millisecond interval timer and auditory reaction time programs for the IBM PC. Behavior Research Methods, Instruments, \& Computers, 19, 30-35.

Nunnally, J. (1978). Psychometric theory (2nd ed.). New York: McGraw-Hill.

Smith, B., \& Puckett, T. (1984, April). Life in the fast lane. PC Tech Journal, pp. 63-74.

STRACK, S. (1987). Development and validation of an adjective checklist to assess the Millon personality types in a normal population. Journal of Personality Assessment, 51, 572-587.

Wigrins, J. S., \& PINCus, A. L. (1989). Conceptions of personality disorders and dimensions of personality. Psychological Assessment: A Journal of Consulting \& Clinical Psychology, 1, 317-322.

\section{NOTES}

1. The term is from Latin, meaning "of the person."

2. Spectra Publishing, 1030D East Duane Ave., Sunnyvale, CA 94086

3. Microsoft Corporation, 16011 NE 36th Way, Box 97017, Redmond, WA 98073-9717.

(Manuscript received July 24, 1989 ; revision accepted for publication May 21, 1990.) 